\title{
A LATTICE THEORETIC CHARACTERIZATION OF AN INTEGRAL OPERATOR 1
}

\author{
L.AWRENCE LESSNER
}

ABSTRACT. We are concerned here with obtaining necessary and sufficient conditions for a linear operator, $K: \mathfrak{l}\left(\mathrm{X}_{1}, \mathbb{Q}_{1}, \mu_{1}\right) \rightarrow$ $M\left(\mathbf{X}_{2}, \mathbb{Q}_{2}, \mu_{2}\right)$, to be represented by an integral, $K(f)=\int k(x, y) f(y) d y$, with an $\mathbb{Q}_{2} \times \mathbb{Q}_{1}$ measurable kernel $k(x, y)$. That such conditions are developed in a lattice the oretic context will be shown to be quite natural. Our direction will be to characterize an integral operator by its action pointwise: i.e., $K(\cdot)(x)$ is a linear functional on a subspace of the essentially bounded functions. Such a development leads one to define the kernel, $k(x, y)$, in a pointwise fashion also, and as a result we are confronted with the question of the $\mathbb{Q}_{2} \times \mathbb{Q}_{1}$ measurability of $k(x, y)$.

Definitions and notation. The following definitions, unless otherwise noted, may be found in [1] and [2].

Definition. The real vector space $R$ is called an ordered vector space when $R$ is partially ordered by $\leq$ and satisfies for $x, y, z \in R$,

(1) $x \leq y$ implies $x+z \leq y+z$,

(2) $x \geq 0$ implies $r x \geq 0$ for any real number $r \geq 0$.

The ordered vector space $R$ is called a Riesz space when for each $x, y \in$ $R$ the least upper bound of $x$ and $y$, written $x \vee y$, exists in $R$. The Riesz space $(R, \leq)$ is called Dedekind complete if for any subset $\left\{x_{a}\right\} \subset R$ such that there is an upper bound $y \in R$ for $\left\{x_{\alpha}\right\}$, then the least upper bound of $\left\{x_{a}\right\}$ exists in $R$ : written $\sup \left\{x_{a}\right\} \in R$. A sequence $\left\langle x_{n}\right\rangle$ on $R$ is said to converge in order to $x \in R$, written $x_{n} \rightarrow x(0)$, whenever $\underline{\lim }_{n}=\varlimsup_{\lim x_{n}}=$ $x$ where

$$
\underline{\lim } x_{n}=\sup _{n} \inf _{k \geq n} x_{k}, \quad \overline{\lim } x_{n}=\inf _{n} \sup _{k \geq n} x_{\dot{k}}
$$

and inf as usual means greatest lower bound. By $0 \leq x_{n} \uparrow x$ we mean $0 \leq$ $x_{n} \leq x_{n+1}$ and $\sup _{n} x_{n}=x ; x_{n} \downarrow x$ means $x_{n} \geq x_{n+1}$ and $\inf _{n} x_{n}=x$. A linear mapping $T: R_{1} \rightarrow R_{2}$ between Riesz spaces is called (0)-continuous when it maps order convergent sequences into order convergent sequences, and $T$ is called positive when $x \geq 0$ implies $T(x) \geq 0$.

\footnotetext{
Received by the editors November 10, 1972 and, in revised form, March 12, 1973. AMS (MOS) subject classifications (1970). Primary 47A65, 47B55, 47C99, 47015, 46G15.

Key words and phrases. Integral operator, lift, Riesz space.

1 This work is part of a doctoral thesis written under the direction of $\mathrm{D}_{\mathrm{r}}$. Alan Schumitzky at the University of Southern California.
} 
Let $(\mathbf{X},(\mathfrak{A}, \mu)$ be a $\sigma$-finite measure space and denote by $M(\mathbf{X})$ the collection of all equivalence classes of ( $\mathcal{1}$ measirable finite $\mu$ a.e. real valued functions on $\mathbf{X}$ modulo $\mu$ null functions. For $f, g \in M(\mathbf{X})$ define $f \leq g$ providing $f(x) \leq g(x)$ for $\mu$ a.e. $x \in \mathbf{X}$, then $(M(\mathbf{X}), \leq)$ is a Dedekind complete Riesz space: see $[1$, p. 126] or $[3$, p. 335]. A linear subspace $\mathscr{C} \subset M(\mathbf{X})$ is called an ideal when $g \in M(X), f \in \mathcal{L}$ and $|g| \leq|f|$ implies $g \in \mathfrak{Q}$. We say that a sequence $\pi=\left\langle x_{j}\right\rangle$ is admissible for $\ell^{P}$ when $\left\{x_{j}\right\}$ is a countable collection of disjoint, measurable sets of finite measure where $\boldsymbol{U}_{x_{j}}=\mathbf{X}$ and $\chi\left(x_{j}\right) \in \mathcal{P}$. For a set $E, \chi(E)$ is the characteristic function of $E$. We shall be required to distinguish between equivalence classes of functions and functions which are defined and finite everywhere. Let $\psi$ be the canonical homomorphism that sends a function $f$, defined and finite real valued a.e., to its equivalence class $\langle f\rangle: \psi(f)=\langle f\rangle$. For $S \subset M(\mathbf{X})$, let $\bar{S}=\{f \mid f: \mathbf{X} \rightarrow$ $(-\infty, \infty)$ and $\psi(f) \in S\}$. We may partially order $\overline{M(X)}$ as follows: for $f, g \in$ $\overline{M(X)}, f \leq g$ if and only if for all $x \in \mathbf{X}, f(x) \leq g(x)$. If $S$ is an ideal of $M(\mathbf{X})$, then $\bar{S}$ is an ideal of $\overline{M(\mathbf{X})}$. Although $M(\mathbf{X})$ is Dedekind complete, in general $\overline{M(X)}$ is not Dedekind complete. For $L$ an ideal of $M(\mathbf{X}), f_{n}, f \in \bar{L}$, we have $f_{n} \rightarrow f(0)$ in $\bar{L}$ when there exists $g \in \bar{L}$ such that for all $n$ and all $x \in X$, $\left|f_{n}(x)\right| \leq g(x)$ and $\lim _{n}(x)=f(x)$ [2, p. 64].

For the duration of this paper we shall assume that $\left(X_{1}, \mathcal{G}_{1}, \mu_{1}\right)$ and $\left(\mathbf{X}_{2},\left(\mathbb{f}_{2}, \mu_{2}\right)\right.$ are $\sigma$-finite measure spaces with respect to nonnegative, countably additive, extended real valued set functions $\mu_{1}$ and $\mu_{2}$, respectively. We also assume that $\left(\mathbf{X}_{1}, \mathbb{G}_{1}, \mu_{1}\right)$ is a separable measure space. We shall denote by $\mathcal{L}$ an ideal of $M\left(\mathbf{X}_{1}\right)$ with an admissible sequence $\pi=\left\langle X_{j}\right\rangle$.

Let $T: \mathcal{L} \rightarrow M\left(\mathbf{X}_{2}\right), S$ a linear subspace of $\mathcal{Q}$ and $\hat{T}: S \rightarrow \overline{M\left(\mathbf{X}_{2}\right)}$; then $\hat{T}$ is called a lift of $T$ on $S$ when for all $f \in S, \psi \circ \hat{T}(f)=T(f)$. If a lift $\hat{T}$ of $T$ on $S$ exists, it need not be unique, also $\hat{T}$ need not inherit even the simplest properties of $T$ : linearity, positivity, order continuity.

A map $K: P \rightarrow M\left(\mathbf{X}_{2}\right)$ is called an integral operator when there is $k(x, y)$ $\in M\left(\mathbf{X}_{2} \times \mathbf{X}_{1}\right)$ such that for $f \in \mathcal{L}$,

$$
K(f)(x)=\int k(x, y) f(y) d \mu_{1}(y) .
$$

$k$ is called the kernel of $K$, and the association is denoted $K=[k]$. That an ideal $\mathcal{L}$ of $M\left(\mathbf{X}_{1}\right)$ with an admissible sequence $\pi$ is a natural domain for an integral operator may be inferred from [4] and [7]. For each $f \in \mathscr{Q}$ and a.e. $x \in X_{2}$ fixed, $k(x, y) f(y)$ is integrable on $X_{1}$; so for a.e. $x$,

$$
\int|k(x, y)| f(y) d \mu_{1}(y)<\infty
$$

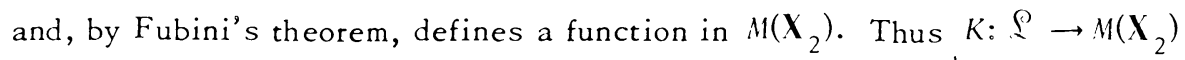
can be considered the difference of two positive operators $\left[k^{+}\right],\left[k^{-}\right]$: i.e.,

$$
K(f)=\int k^{+}(x, y) f(y) d y-\int k^{-}(x, y) f(y) d y
$$


where $\left[k^{ \pm}\right]: \mathfrak{C} \rightarrow M\left(\mathbf{X}_{2}\right)$ are positive operators.

The following theorem gives necessary and sufficient conditions for an operator $T: \mathcal{Q} \rightarrow M\left(\mathbf{X}_{2}\right)$ to be an integral operator. Since an integral operator is necessarily the difference of two positive operators, it will suffice to consider positive operators only.

Theorem. Let $T: \mathcal{L} \rightarrow M\left(\mathbf{X}_{2}\right)$ be a positive linear operator; then there exists $k \in M\left(\mathbf{X}_{2} \times \mathbf{X}_{1}\right)$ such that $T$ is an integral operator, $T=[k]$ and 0 $\leq k$ if and only if

(1) $T: \mathscr{P} \rightarrow M\left(\mathbf{X}_{2}\right)$ is (0)-continuous,

(2) for each $j$ there is a lift $\hat{T}_{j}$ of $T$ on $L_{\infty}\left(x_{j}\right)$ that is positive, linear and order continuous.

Proof. Let $T=[k]$ be a positive linear integral operator with kernel $0 \leq k_{0}$ By $[2, \mathrm{p} .215]$, it is sufficient to show that if $f_{n} \in \mathcal{Q}$ and $0 \leq f_{n} \downarrow 0$, then $0 \leq T\left(f_{n}\right) \downarrow 0$, to obtain order continuity for $T$. This follows easily from the Lebesgue dominated convergence theorem. To verify (2) let $k_{0}$ be a specific kernel: $k_{0} \in k \cap \overline{M\left(\mathbf{X}_{2} \times \mathbf{X}_{1}\right)}$ such that $0 \leq k_{0}(x, y)$ for every $(x, y)$ $\epsilon \mathbf{X}_{2} \times \mathbf{X}_{1}$. For each $j, \int k_{0}(x, y) \chi\left(x_{j}\right)(y) d y<\infty$ except for $x \in A_{j}$ where $\mu_{2}\left(A_{j}\right)=0$. Now take $k_{1}(x, y)=k_{0}(x, y)$ for $(x, y) \in \widetilde{\mathcal{U}_{A}}{ }_{j} \times X_{1}$ and define $k_{1}(x, y)=0$ for $(x, y) \in \bigcup A_{j} \times X_{1}$. Thus $k_{1}$ and $k_{0}$ differ on a set of $\mu_{2}$ $\times \mu_{1}$ measure zero. If $f \in L_{\infty}\left(x_{j}\right)$, then for some $0<c$ we have $|f| \leq c \chi\left(x_{j}\right)$. Consequently,

$$
\left|\int k_{1}(x, y) f(y) d y\right| \leq \int k_{1}(x, y) \cdot c \chi^{\left(x_{j}\right)(y) d y<\infty}
$$

for all $x \in \mathbf{X}_{2}$. For $f \in L_{\infty}\left(x_{j}\right)$, define

$$
\hat{T}_{j}(f)=\int k_{1}(x, y) f(y) d y
$$

then $\hat{T}_{j}$ is a lift of $T$ on $L_{\infty}\left(x_{j}\right)$. It is obvious that $\hat{T}_{j}$ is positive and linear. If $f_{n} \in L_{\infty}\left(x_{j}\right), 0 \leq f_{n} \downarrow 0$, then a simple application of Lebesgue's dominated convergence theorem shows that $0 \leq \hat{T}_{j}\left(f_{n}\right)(x) \downarrow 0$ for all $x$ and $\hat{T}_{j}$ is order continuous.

Now let us suppose that $T: \mathcal{L} \rightarrow M\left(\mathbf{X}_{2}\right)$ is a positive, linear and order continuous map that satisfies (2). We shall construct the integral representation for $T$ by viewing " $\hat{T}_{j}()(x)$ " as a measure on the relativized space $\left(X_{j},\left(\mathscr{A}_{1} \cap X_{j}, \mu_{1}\right)\right.$ and applying the Radon-Nikodym theorem. Let $\mathbb{C}_{j}=$ $\left\{E \cap X_{j}: E \in \mathcal{G}_{1}\right\}$ and $x \in X_{2}$ be fixed, then define for $E \in \mathbb{G}_{j}, \mu_{x_{j}}(E)=$ $\hat{T}_{j}(\langle\chi(E)\rangle)(x)$. The finite additivity of $\mu_{x_{j}}$ follows from the linearity of $\hat{T}_{j}$, and the nonnegativity of $\mu_{x_{j}}$ comes from the positivity of $\hat{T}_{j}$. Let $\left\langle E_{i}\right\rangle$ be a countable disjoint sequence from $\mathbb{G}_{j}$; then $\chi\left(\bigcup_{i=1}^{\infty} E_{i}\right)-\chi\left(\bigcup_{i=1}^{n} E_{i}\right)_{i}=f_{n}$ and $0 \leq f_{n} \downarrow 0$. Since $\hat{T}_{j}$ is order continuous, $0 \leq \hat{T}_{j}\left(f_{n}\right)(x) \downarrow 0$ for all $x \in \mathbf{X}_{2}$. Consequently, $\lim _{n \rightarrow \infty} \mu_{x_{j}}\left(\bigcup_{1}^{\infty} E_{i}\right)-\sum_{i=1}^{n} \mu_{x_{j}}\left(E_{i}\right)=0: \mu_{x_{j}}$ is countably additive. 
Now if $E \in \mathbb{C}_{j}$ is a $\mu_{1}$ null set, then

$$
\hat{T}_{j}(\langle\chi(E)\rangle)(x)=\hat{T}(2\langle\chi(E)\rangle)(x)=2 \hat{T}(\langle\chi(E)\rangle)(x)
$$

for all $x \in \mathbf{X}_{2}$, so $\hat{T}_{j}\left(\left\langle\left\langle_{\chi}(E)\right\rangle\right)(x)=0\right.$ for all $x \in \mathbf{X}_{2}$ and $\mu_{x_{j}}$ is absolutely continuous with respect to $\mu_{1}$. Let $k_{j}(x, y)$ be the Radon-Nikodym derivative of $\mu_{x_{j}}$ with respect to $\mu_{1}$. Clearly $0 \leq k_{j}(x, y)$ for all $x$ and $y$, Since $\left(\mathbf{X}_{1}, \mathbb{A}_{1}, \mu_{1}\right)$ is separable, it follows by $[5, \mathrm{p} .616]$, that $k_{j}(x, y)$ is $\left(\mathbb{A}_{2} \times \mathbb{C}_{1}\right.$ measurable.

For $E \in\left(\mathbb{H}_{j}\right.$ we have $\mu_{x_{j}}(E)=\int k_{j}(x, y) \chi(E)(y) d y$. Thus for any simple function $r=\sum_{i=1}^{n} c_{i} \chi\left(E_{i}\right)$ where $\left\{E_{i}\right\}_{i=1}^{n}$ are disjoint and $E_{i} \subset X_{j}$,

$$
\hat{T}_{j}(\langle r\rangle)(x)=\sum_{i=1}^{n} c_{i} T_{j}\left(\left\langle\chi\left(E_{i}\right)\right\rangle\right)(x)=\sum_{i=1}^{n} c_{i} \mu_{x_{j}}\left(E_{i}\right)=\int k_{j}(x, y) r(y) d y
$$

for all $x \in \boldsymbol{X}_{2}$. Thus

$$
T(r)-v^{\prime} \circ k_{j}(x, y) r(y) d y
$$

for any simple function $r$ vanishing outside $X_{j}$,

Now let $0 \leq f \in \mathcal{Q}^{\mathcal{D}}$ and $f$ vanish off of $X_{i}$; then there exists a sequence $\left\langle f_{n}\right\rangle$ of simple functions such that $0 \leq f_{n} \uparrow f$ and $f_{n}$ vanishes off of $X_{j}$. So

$$
T(f)=\lim _{n \rightarrow \infty} T\left(f_{n}\right)=\lim _{n \rightarrow \infty} \dot{\int} \int k_{j}(x, y) f_{n}(y) d y=\psi \circ \int k_{j}(x, y) f(y) d y .
$$

If we drop the distinction between equivalence classes and functions, we now have for all $f \in \mathbb{P}$ that vanish off of $X_{j}$ that $T(f)=\int k_{j}(x, y) f(y) d y$. The extension from nonnegative $f$ to general $f$, as usual, uses the decomposition $f=f^{+}-f^{-}$where $f^{+}=f \vee 0$ and $f^{-}=-f \vee 0$.

If we set $k=\sum_{j=1}^{\infty} k_{j}$, then $0 \leq k$, and $k$ is $\mathbb{A}_{2} \times \mathbb{A}_{1}$ measurable. Since $k \chi\left(x_{j} \times X_{1}\right)=k_{j}, k<\infty, \mu_{2} \times \mu_{1}$ a.e. Now let $0 \leq f \in \mathcal{Q}^{\infty}$ and $f=\sum_{j=1}^{\infty} f_{j}$ where $0 \leq f_{j}=f \cdot \chi\left(x_{j}\right)$. Since $0 \leq \sum_{j=1}^{n} f_{j} \uparrow f$.

$$
\begin{aligned}
T(f) & =\sum_{j=1}^{\infty} T\left(f_{j}\right)=\sum_{j=1}^{\infty} \int k_{j}(x, y) f(y) d y \\
& =\sum_{j=1}^{\infty} \int k(x, y) f(y) \chi\left(x_{j}\right)(y) d y=\int k(x, y) f(y) d y .
\end{aligned}
$$

The extension from nonnegative $f$ to general $f \in \stackrel{(}{*}$, as before, uses $f=f^{+}$ $-f^{-}$.

In this paragraph we provide an example of a lift of a rather well-known operator. Let $(\mathbf{X}, \mathbf{C}, \mu)$ be the usual Lebesgue measure on the real line $\mathbf{X}$, and let $L_{2}$ be the square integrable functions defined on $\mathbf{X}$. Clearly $L_{2}$ is an ideal of $M(\mathbf{X})$ having an admissible sequence. An integral operator $K$ : $L_{2} \rightarrow L_{2}$, where $K=[k]$ is of Hilbert-Schmidt class when $\iint|k(x, y)|^{2} d x d y$ $<\infty$ : see [8]. Let us suppose $0 \leq k$, and choose any specific representative 
$k_{1}$ of $k_{\text {. So }} k_{1}$ is square integrable and there is a $\mu$ null set $A$ such that if $x \notin A$, then $0 \leq \int k_{1}(x, y) f(y) d y<\infty$. Now define $k_{0}(x, y)=k_{1}(x, y)$ when $x \notin A$ and $k_{0}(x, y)=0$ when $x \in A$. Thus $k_{1}-k_{0}$ is $\mu \times \mu$ null function and $K=\left[\left\langle k_{0}\right\rangle\right]$. We may now define a lift $\hat{K}$ of $K$ on $L_{2}$ by $\hat{K}(f)(x)=$ $\int k_{0}(x, y) f(y) d y$ : i.e. for all $f \in L_{2}$ and for all $x \in \mathbf{X}, 0 \leq \int k_{0}(x, y) f(y) d y$ $<\infty$.

\section{BIBLIOGRAPHY}

1. W. A. J. Luxemburg and A. C. Zaanen, Riesz spaces, North-Holland, Amsterdam, 1971.

2. B. Z. Vulih, Introduction to the theory of partially ordered spaces, Fizmatgiz, Moscow, 1961; English transl., Noordhoff, Groningen, 1967. MR 24 \#A3494; 37 \#121.

3. N. Dunford and J. T. Schwartz, Linear operators. I. General theory, Pure and Appl. Math., vol. 7, Interscience, New York, 1958. MR 22 \#8302.

4. N. Aronszajn and P. Szeptyski, $O_{n}$ general integral transformations, Math. Ann. 163 (1966), 127-154. MR 32 \#8209.

5. J. L. Doob, Stochastic processes, Wiley, New York; Chapman \& Hall, London, 1953. MR 15, 445.

6. A. Ionescu Tulce a and C. Ionescu Tulcea, Topics in the theory of lifting, Ergebnisse der Mathematik und ihrer Grenzgebiete, Band 48, Springer-Verlag, New York, 1969. MR $43 \# 2185$.

7. W. A. J. Luxemburg and A. C. Zaanen, The linear modulus of an order bounded linear transformation. II, Nederl. Akad. Wetensch. Proc. Ser. A75 = Indag. Math. 34 (1972).

8. A. C. Zaanen, Linear analysis, North-Holland, Amsterdam, 1964.

DEP ARTMENT OF MATHEM ATICS, ARYA-MEHR UNIVERSITY OF TECHNOLOGY, TEHRAN, IRAN 